\title{
NEW CONCEPT OF A PRESSURE BALANCED VARIABLE DISPLACEMENT VANE PUMP
}

\author{
Arie Perry Ph. D. \\ Israeli Aircraft Industries Ltd. - MLM Division \\ Beer Yaakov, P.O. Box 45, 70350 ISRAEL \\ Tel: 972 - 8 - 9272496; Fax: 972 - 8 - 9272890 \\ E-Mail : aperry@mlm.iai.co.il
}

\begin{abstract}
Vane pumps come in two basic forms: pressure balanced, fixed displacement and pressure unbalanced, variable displacement. In an unbalanced variable displacement vane pump, vanes sweep against an adjustable cam ring. Moving the ring against a spring changes the radial travel of the vanes, varying pump displacement. This design imposes side loads on the bearings and bending moments on the shaft, limiting the pump operating pressure range and its possible applications.

We introduce a New Concept of a Pressure Balanced Variable Displacement Vane Pump. In the new concept the vane pump has a thin ring installed concentric with the rotor. To vary pump displacement, two equal opposed forces are applied to the ring (vertical), in effect "squeezing" it to an elliptical shape. The displacement is controlled by the amount of out-of-round ring deflection, the greater the squeeze, the greater the ellipse, the greater the flow output.

The stresses in the ring is kept well within the elastic range of the ring material, so when the deflecting forces are removed, the ring returns to its circular shape and displacement returns to zero. Now, applying the forces along the (horizontal) diameter will exchange suction-pressure ports tasks, in effect, making the pump bi-directional. The new pump has a fast response, and incorporating such a pump in servo systems, would make the basis for a fully hydrostatic low cost vane type hydraulic pump system.
\end{abstract}

\section{KEY WORDS}

Pressure Balanced Variable Displacement Vane Pump 


\section{NOMENCLATURE}

a : $\quad$ Ring ellipse big axis

b : $\quad$ Ring ellipse small axis

$\Delta \mathrm{b}$ : Change in small axis

D : $\quad$ Pump rotor diameter

Dx : Horizontal Ring deflection

Dy : Vertical Ring deflection

E : Modulus of elasticity

h : $\quad$ Pump thickness

I : $\quad$ Ring moment of inertia

n : $\quad$ Pump RPM

$\mathrm{P}: \quad$ Applied force

$\mathrm{R}$ : $\quad$ Ring radius

Q : $\quad$ Pump displacement

$\Delta \mathrm{Q}$ : $\quad$ Change in displacement

$\delta$ : $\quad$ Ring thickness

$\sigma: \quad$ Stress in the ring

\section{INTRODUCTION}

Present day variable displacement vane pumps are based on the principle of the eccentric ring. The result is an unbalanced pressure, causing radial loads on the bearings and bending moments on the shaft, thus limiting maximum pump operating pressure.

Today's fixed displacement pumps are all pressure balanced, base on the elliptic contour, forming two suction (low pressure) and two exhaust (high pressure) symmetric vents. Change (increase) of pump displacement is possible only by replacing pump housing with a contour of different elliptic dimensions.

In the paper we introduce a New Concept of a Pressure Balanced Variable Displacement Vane Pump. We bring here the highlights of the design considerations and the mathematical calculations of the new pump displacement.

A pump prototype was built during the development process, and pressure, volumetric and efficiency tests made, showing beyond any dought concept feasibility. The new concept is introducing an inexpensive bi-directional fast response pump, competitive with the costly piston type pumps used today in servo systems.

\section{PRINCIPLE OF OPERATION}

In the new concept, the vane pump has a thin wall ring $(2 \mathrm{~mm})$ installed concentric with the rotor. To vary pump displacement, two equal opposed forces are applied (vertically) to the ring in effect "squeezing" it to an elliptical shape. Displacement is controlled by the amount of out-of-round ring deflection, the greater the squeeze, the greater the ellipse, the greater the flow output, see Fig. 1. A change of 3-4 $\mathrm{mm}$ in ring roundness causes a flow change from zero to maximum, and. a change of $0.2 \mathrm{~mm}$ for example, causes a 1 lit/min pump flow rate change.

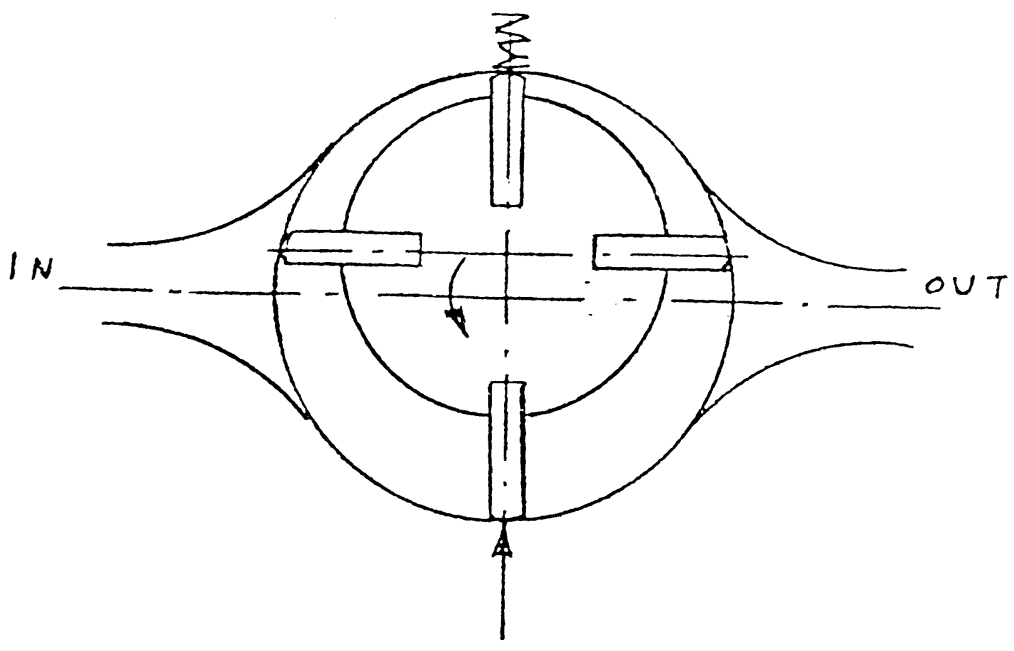

Figure 1 - Pump principle of operation

The ring is made of a material with low module of elasticity and high surface hardness. The stresses in the ring, during deflection, are kept within the elastic range, therefor when deflecting forces are released, ring returns to concentricity and pump displacement zeros. 


\section{Geometrical limitations}

When designing the thin ring diameter for a given pump rotor, there are two geometrical limitations for the small and big axis of the possible attainable ellipse, see Figure (2)

- Ring small axis (b) has to be bigger than the rotor diameter (D).

\section{$2 b>D$ rotor}

- Ring big axis (a) must not exceed rotor diameter (D) plus the length of the vane (1).

\section{$2 \mathrm{a} \leq \mathrm{D}$ rotor $+\mathrm{I}$ vane}

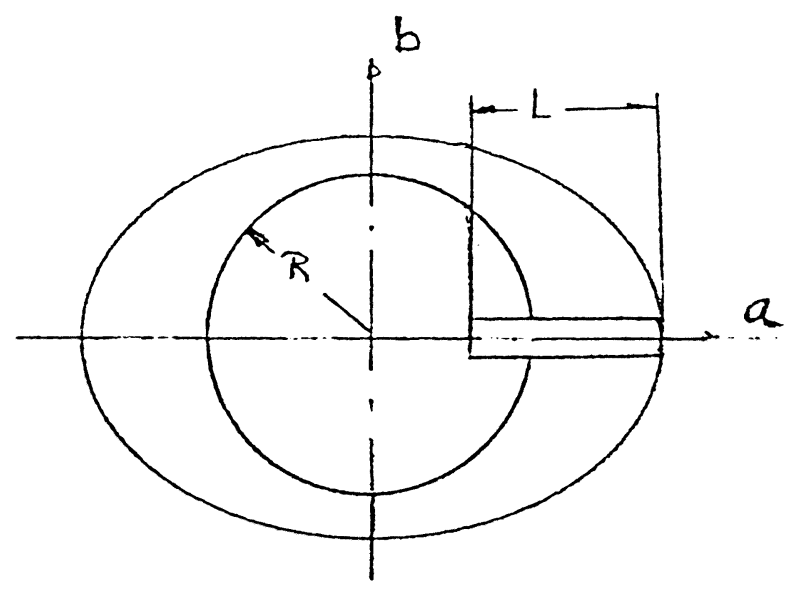

Figure 2 -Geometrical limitations

\section{Material limitations}

There are three force sources on the ring:

- Vane centrifugal forces - negligible

- Oil pressure - non existent, since the pump is fully pressure balanced

- Deflection bending moments - dominant

The bending moment in the ring as function of location $(\Theta)$ is given in $\mathrm{Eq}(1)$

$M=\frac{P R}{2}\left(\frac{2}{\pi}-\sin \theta\right)$

And the maximum occurs at $\Theta=0$, Eq. (2)

$\mathbf{M}=\frac{\mathbf{P R}}{2}$

Deflection in the ring is given in Eq. (3) \&(4)

$$
\begin{aligned}
& D x=0.137 \frac{P R^{3}}{E I} \ldots \\
& D_{y}=-0.149 \frac{P R^{3}}{E I}
\end{aligned}
$$

Combining Eq. (2) \& (3), we get the stress on the ring as function of; module of elasticity, ring thickness \& radius, and ring deflection, as given in Eq.(5)

$$
\sigma=\frac{\mathbf{E}}{\mathbf{R}^{2}} \mathbf{D y} \delta
$$

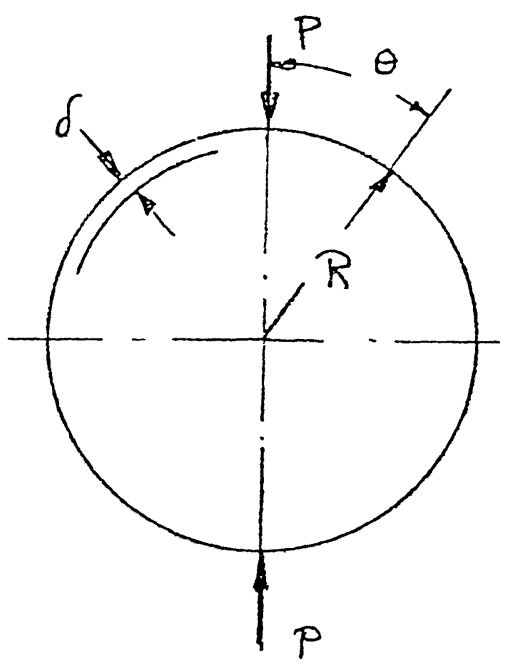

Figure 3 - Stresses on the ring

As one can see, in order to keep the stress in the ring in the elastic range, we have to design the ring within the following constrains:

- Maximum diameter (2R) in accordance to the geometrical limitations

- Ring deflection (Dy) is also geometrically limited, but has to be as great as possible for maximum attainable flow rate

- Material choice with a low (E) modulus of elasticity

- Ring thickens $(\delta)$ about $2 \mathrm{~mm}$, the practical manufacturing possibility 


\section{THE NEW PUMP}

The new pump was developed based on a standard commercial vane pump. A proof-ofconcept-prototype was built and tested to prove the concept. We replaced the main part (housing) of the pump and the ring, Fig. (4).

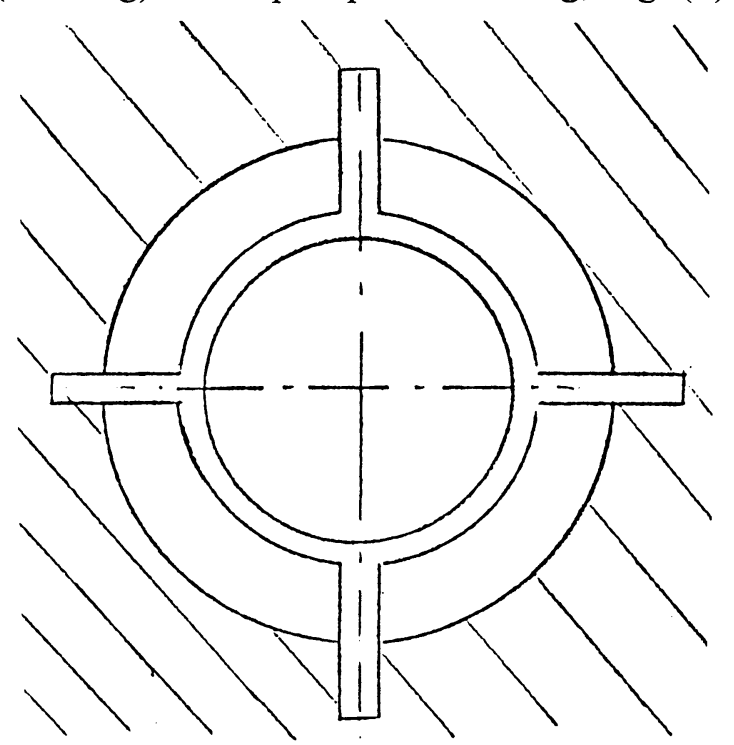

Figure 4 - The new concept

\section{New Pump Displacement}

The change in volume in the pump, Eq. (6)(7)

$$
\mathbf{V}_{\rho}=\frac{\mathbf{d V}}{\mathbf{d f}}
$$

Where: $\quad x=\cos p: y=\sin p$

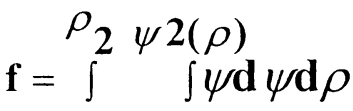

$$
\begin{aligned}
& \rho_{1} \psi 1(\rho)
\end{aligned}
$$

After this integration we get: Eq. (8)

$$
\mathrm{V}_{\rho}=\frac{1}{2} \mathrm{~h}\left(\psi_{\mathrm{k}}^{2}-R_{\mathrm{k}}^{2}\right)
$$

In case of 12 vanes we get: Eq. (9)

$$
\begin{aligned}
& \mathbf{W}=\pi \mathbf{h}\left(\mathbf{a}^{2}-\mathbf{R}_{\mathrm{k}}^{2}\right) \\
& \mathbf{a}^{2}=2 \mathbf{R}^{2}-\mathbf{b}^{2} \text { and } \max \text { when } \mathbf{b}=\mathbf{R}_{k}
\end{aligned}
$$

The change in displacement as a function of ring deflection: Eq. (10) and Qmax: Eq. (11)

$\Delta Q=2 \pi h \mathbf{n} \mathbf{b} \Delta \mathbf{b}$

$\mathbf{Q m a x}=2 \pi \mathbf{h} \mathbf{n}\left(\mathbf{R}^{2}-\mathbf{b}^{2}\right)$

And finally, the bending moment on the shaft is not dependent on ring deflection but on pump pressure only: Eq. (12)

$$
M t=2 \pi h \Delta P\left(R^{2}-R_{k}^{2}\right)
$$

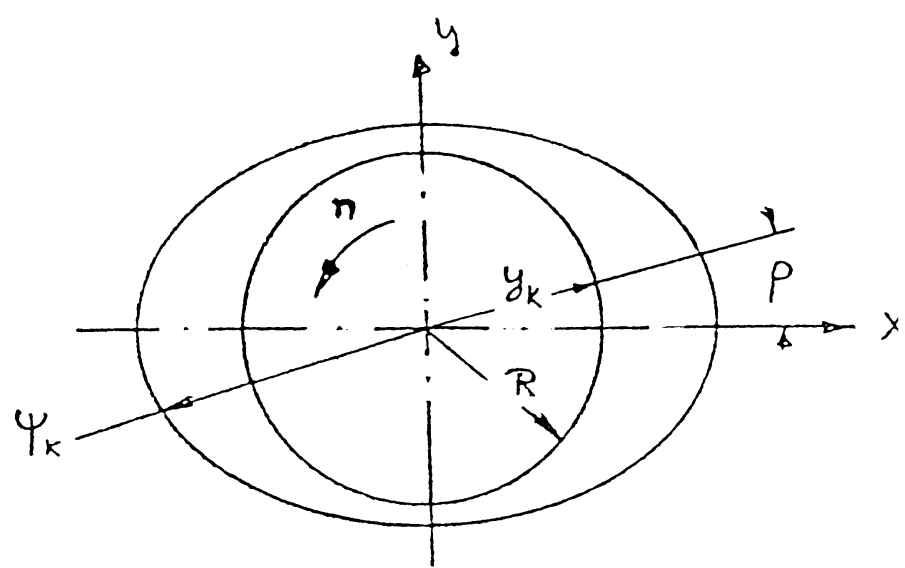

Figure 5-New concept calculation

\section{SUMMARY}

The paper introduced a New Concept for a Variable Displacement Pressure Balanced Vane Pump. The new fast response pump, if adopted by the hydraulic industry, can be the basis for bi-directional, vane type, low cost, fully hydrostatic pump control system.

\section{REFERENCES}

1. - A Theoretical and Experimental Study of Hydraulic Power Supplies. (Dissertation) By: A. Perry -1983 - University Micro. Intl.

2. - Hydraulic Control Systems.

By: H.E. Merritt -1967-Wiley \& Sons Inc. 